\title{
Association between triglyceride glucose index and arterial stiffness in Korean adults
}

\author{
Sang Bae Lee ${ }^{1,2}$, Chul Woo Ahn 1,2, Byoung Kwon Lee ${ }^{3}$, Shinae Kang 1,2, Ji Sun Nam 1,2, Ji Hong You 1,2, \\ Min Jin Kim ${ }^{1,2}$, Min Kyung Kim ${ }^{4}$ and Jong Suk Park ${ }^{1,2^{*}}$
}

\begin{abstract}
Background: The triglyceride glucose (TyG) index has been suggested as a simple surrogate marker of insulin resistance. However, there are limited data regarding the association between the TyG index and arterial stiffness in adults. Therefore, we evaluated the relationship between the TyG index and arterial stiffness as measured based on brachial ankle pulse wave velocity (baPWV) in Korean adults.

Methods: A total of 3587 subjects were enrolled in this study. Anthropometric and cardiovascular risk factors were measured. The TyG index was calculated as In[fasting triglycerides $(\mathrm{mg} / \mathrm{dl}) \times$ fasting glucose $(\mathrm{mg} / \mathrm{dl}) / 2]$, and the insulin resistance index of homeostasis model assessment (HOMA-IR) was estimated. Arterial stiffness was determined by measuring baPWV.

Results: The subjects were stratified into four groups based on the TyG index. There were significant differences in cardiovascular parameters among the groups; the mean baPWV increased significantly with increasing TyG index. According to the logistic regression analysis after adjusting for multiple risk factors, the odds ratio $(95 \% \mathrm{Cl})$ for increased baPWV (> 75th percentile) for the highest and lowest quartiles of the TyG index was 2.92 (1.92-4.44) in men and 1.84 (1.15-2.96) in women, and the odds ratio for increased baPWV for the highest and lowest quartiles of the HOMA-IR was 1.80 (1.17-2.78) in men and 1.46 (1.06-2.47) in women, respectively.
\end{abstract}

Conclusion: The TyG index is more independently associated with increased arterial stiffness than HOMA-IR in Korean adults.

Keywords: Triglyceride glucose index, Arterial stiffness, Pulse wave velocity, Insulin resistance

\section{Background}

Cardiovascular disease (CVD) is a leading cause of death worldwide [1]. In particular, arterial stiffness as measured by brachial-ankle pulse wave velocity (baPWV) has been established as an independent predictor of not only cardiovascular events but also cardiovascular mortality [2].

Insulin resistance (IR) is one of the most important risk factors for CVD [3]. IR is associated with obesity,

\footnotetext{
*Correspondence: PJS00@yuhs.ac

1 Division of Endocrinology, Department of Internal Medicine, Yonsei University College of Medicine, Gangnam Severance Hospital, 211, Eonju-ro, Gangnam-gu, Seoul, South Korea

Full list of author information is available at the end of the article
}

hypertension, and dyslipidemia, all of which predispose individuals to atherosclerosis and CVD [4]. Indeed, previous studies have demonstrated an independent association between IR and cardiovascular events $[5,6]$.

Many recent studies have shown that the triglyceride glucose (TyG) index is associated with IR, as assessed by hyperinsulinemic euglycemic clamp testing and HOMAIR. Thus, the TyG index has been proposed as a reliable and simple surrogate marker of IR [7-10].

Consistent with these data, there is growing evidence to suggest that the TyG index is associated with cardiovascular disease [11-13]. However, few studies have examined the relationship between the TyG index and subclinical atherosclerosis $[11,14,15]$. Moreover, to the 
best of our knowledge, there has to date only been one study, which was performed in a small population of women, to investigate the relationship between the TyG index and arterial stiffness [16]. Therefore, in the present study, we investigated the relationship between the TyG index and arterial stiffness and compared the data with that of HOMA-IR in Korean adults.

\section{Methods}

\section{Study population}

The study population consisted of 5989 Korean subjects who participated in a comprehensive health examination as part of a self-referred health checkup program at the Gangnam Severance Hospital Health Promotion Center from January 2008 to February 2015. The exclusion criteria of this study were: subjects with elevated triglyceride levels $(>400 \mathrm{mg} / \mathrm{dl})$ and subjects with any history of malignancy, acute inflammatory disease, infectious disease, or renal disease. We also excluded subjects with a history of hypertension, angina, myocardial infarction, cerebrovascular accident, or an ankle-brachial index $(A B I)<0.90$. Subjects taking statins or triglyceride-lowering medications (fenofibrate or omega-3) were also excluded. In addition, we excluded men and women with a history of alcohol consumption in excess of 30 and $20 \mathrm{~g} /$ day, respectively. After applying the exclusion criteria, a total of 3587 subjects were enrolled in our final analysis. The study protocol was approved by the Institutional Review Board of Yonsei University College of Medicine.

\section{Clinical characteristics}

We measured the height and weight of each study participant and calculated the body mass index (BMI, kg/ $\mathrm{m}^{2}$ ). Lifestyle information, personal medical history of acute and chronic illnesses, and medication history were collected using a standard questionnaire. Systolic and diastolic blood pressures (SBP, DBP) were measured by experienced technicians using an automated blood pressure (BP) monitor (HEM-7080IC; Omron Healthcare, Lake Forest, IL, USA) after a 5-min rest period with the patient's arm placed at heart level. The diagnosis of diabetes mellitus was based on a previous history of diabetes or the ADA's diagnostic guidelines. Hypertension was defined as systolic and/or diastolic blood pressures $>140 / 90 \mathrm{mmHg}$ or the current use of an antihypertensive medication. Current smokers were defined as having smoked cigarettes regularly over the previous 6 months.

\section{Biochemical parameters}

Blood samples were obtained from all subjects after $8 \mathrm{~h}$ of fasting. Samples were immediately centrifuged, and the serum samples were stored at $-70{ }^{\circ} \mathrm{C}$ until analysis. The fasting plasma glucose (FPG), total cholesterol
(TC), high-density lipoprotein cholesterol (HDL-C), and triglycerides (TG) were determined using enzymatic methods with a Hitachi 7600-120 automated chemistry analyzer (Hitachi, Tokyo, Japan). Low-density lipoprotein cholesterol (LDL-C) was calculated according to the Friedewald formula. The TyG index was calculated as $\ln$ [fasting triglycerides $(\mathrm{mg} / \mathrm{dl}) \times$ fasting glucose $(\mathrm{mg} /$ dl)/2] [7]. The fasting serum insulin level was determined by chemiluminescence with an RIA kit (Daiichi, Japan). Insulin resistance was estimated using the homeostasis model assessment of insulin resistance (HOMA-IR) index calculated as fasting insulin $(\mu \mathrm{U} / \mathrm{mL}) \times$ fasting plasma glucose $(\mathrm{mmol} / \mathrm{L}) / 22.5$.

\section{Pulse wave velocity}

Arterial stiffness was measured using an automatic plethysmographic instrument (VP-1000; Colin, Komaki, Japan) as previously described [17]. Briefly, electrodes were placed on both wrists, and cuffs were wrapped around both the upper arms and ankles. After simultaneous measurement of blood pressure and waveforms in all four limbs, the time interval between the brachial and ankle waveforms $(\Delta \mathrm{Tba})$ was determined. The distance between the brachium and the ankle $(\mathrm{La}-\mathrm{Lb})$ was estimated automatically according to the subject's height. After these data were collected, baPWV was calculated using the following equation: $\mathrm{baPWV}=(\mathrm{La}-\mathrm{Lb}) / \Delta \mathrm{Tba}$ (in $\mathrm{cm} / \mathrm{s}$ ). Both baPWV values were measured after allowing the patient to rest in a supine position for at least $5 \mathrm{~min}$. The mean of the right and left baPWV was used as a marker of arterial stiffness.

\section{Statistical analysis}

Continuous variables with normal distributions were expressed as the mean \pm standard deviation (SD), whereas continuous variables with skewed distributions were expressed as the medians with interquartile ranges and were $\log$ transformed for analysis. Intergroup comparisons were performed using ANOVA. Chi square tests were used to compare categorical variables with percentages. Age-adjusted baPWV means and standard errors were calculated using analysis of covariance (ANCOVA) according to TyG quartiles. The relationships between the baPWV and various clinical parameters were examined using Pearson's correlation. The odds ratios (OR) and corresponding $95 \%$ confidence intervals (CI) for high PWVs were estimated by multivariate logistic regression analysis after adjusting for confounding variables across TyG index and HOMA-IR quartiles. Covariates in the multivariable model, which were chosen for their clinical importance, as well as statistical significance, were age, SBP, BMI, LDL-C, HDL-C, diabetes mellitus, and menopause in women. Because an absolute cut-off 
value of normal baPWV was not available, high baPWV was arbitrarily defined as a value greater than the cut-off level between the third and fourth quartiles ( $>75$ th percentile), which was $1437.5 \mathrm{~cm} / \mathrm{s}$ for men and $1411.5 \mathrm{~cm} / \mathrm{s}$ for women. All statistical analyses were performed using SPSS for Windows 23.0 (SPSS Inc., Chicago, IL, USA). $\mathrm{P}$ values less than 0.05 were considered statistically significant.

\section{Results}

\section{Baseline characteristics}

Table 1 shows the clinical and laboratory characteristics of the study population. Subjects were stratified into four groups based on their TyG index levels. Significant differences in metabolic parameters were observed among the groups. SBP, DBP, BMI, FPG, TC, TG, LDL-C, and HOMA-IR were all positively associated with the TyG index, while HDL-C was negatively associated with the TyG index. In addition, smoking status and the prevalence of diabetes were positively associated with TyG index quartiles.

We found that baPWV was positively associated with increasing TyG quartiles. Figure 1 shows the age-adjusted mean baPWV according to TyG index quartiles in the overall population, where $\mathrm{Q} 1=1308.9, \mathrm{Q} 2=1338.4$, $\mathrm{Q} 3=1352.9$, and $\mathrm{Q} 4=1387.7 \mathrm{~cm} / \mathrm{s} \quad(\mathrm{P}<0.01)$. For men, the age adjusted mean baPWVs of the TyG index quartiles were $1344.9,1358.2,1360.7$ and $1394.3 \mathrm{~cm} / \mathrm{s}$ $(\mathrm{P}<0.01)$, while for women the age-adjusted mean baPWVs in the four quartiles were 1291.8, 1318.0, 1337.9 and $1361.1 \mathrm{~cm} / \mathrm{s}$, respectively $(\mathrm{P}<0.01)$.

\section{Correlation between the baPWV and clinical variables}

Pearson's correlation analysis was used to examine the relationship between the baPWV and clinical variables. The baPWV was significantly correlated with age SBP, DBP, BMI, TC, LDL-C, HDL-C, TG/HDL-C, LDL-C/ HDL-C, non HDL-C HOMA-IR, and the TyG index (Table 2).

\section{Logistic regression analysis of TyG index, HOMA-IR and high baPWV}

The association between the TyG index and high baPWV was further explored by categorizing the TyG index levels

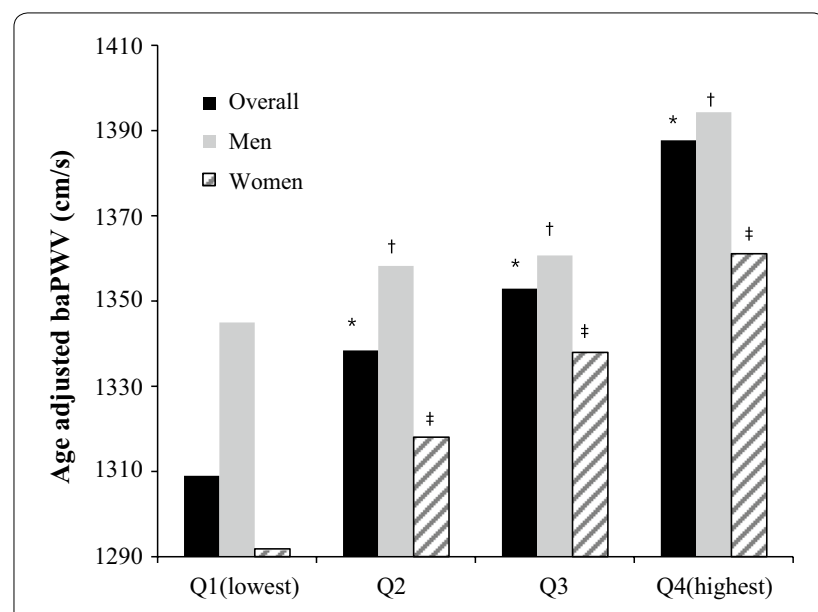

Fig. 1 Age-adjusted mean baPWV for the overall population, men, and women $\left({ }^{*},+\neq \mathrm{P}<0.01\right.$ vs Q1)

Table 1 Clinical characteristics of the study population according to TyG index

\begin{tabular}{|c|c|c|c|c|c|}
\hline & Q1 (lowest) & Q2 & Q3 & Q4 (highest) & $P$ value \\
\hline $\mathrm{N}$ & 897 & 896 & 898 & 896 & \\
\hline Age (years) & $49.91 \pm 9.83$ & $52.88 \pm 8.99$ & $53.30 \pm 8.77$ & $52.03 \pm 8.68$ & $<0.01$ \\
\hline $\operatorname{Sex}(M / F)$ & $286 / 611$ & $433 / 463$ & $601 / 297$ & $741 / 155$ & $<0.01$ \\
\hline $\mathrm{SBP}(\mathrm{mmHg})$ & $114.60 \pm 12.11$ & $117.75 \pm 11.94$ & $120.67 \pm 10.96$ & $121.99 \pm 10.61$ & $<0.01$ \\
\hline DBP $(\mathrm{mmHg})$ & $71.42 \pm 8.33$ & $73.68 \pm 8.03$ & $75.90 \pm 7.18$ & $77.14 \pm 7.23$ & $<0.01$ \\
\hline BMI $\left(\mathrm{kg} / \mathrm{m}^{2}\right)$ & $21.82 \pm 2.65$ & $22.99 \pm 3.02$ & $23.89 \pm 2.78$ & $25.04 \pm 2.69$ & $<0.01$ \\
\hline FPG (mg/dL) & $85.49 \pm 8.92$ & $92.54 \pm 9.97$ & $96.87 \pm 12.59$ & $102.45 \pm 18.17$ & $<0.01$ \\
\hline $\mathrm{TC}(\mathrm{mg} / \mathrm{dL})$ & $183.15 \pm 33.16$ & $192.48 \pm 33.16$ & $193.78 \pm 34.35$ & $200.77 \pm 35.14$ & $<0.01$ \\
\hline $\mathrm{TG}(\mathrm{mg} / \mathrm{dL})$ & $55(48-62)$ & $79(72-88)$ & $111(99-122)$ & $180(153-226)$ & $<0.01$ \\
\hline LDL-C (mg/dL) & $109.47 \pm 29.61$ & $120.93 \pm 30.02$ & $123.14 \pm 30.93$ & $123.30 \pm 31.76$ & $<0.01$ \\
\hline $\mathrm{HDL}-\mathrm{C}(\mathrm{mg} / \mathrm{dL})$ & $58.60 \pm 12.95$ & $53.26 \pm 12.02$ & $47.59 \pm 10.88$ & $42.10 \pm 8.62$ & $<0.01$ \\
\hline TyG index & $7.73 \pm 0.21$ & $8.20 \pm 0.11$ & $8.57 \pm 0.11$ & $9.16 \pm 0.28$ & $<0.01$ \\
\hline HOMA-IR & $0.60(0.41-0.91)$ & $0.87(0.63-1.28)$ & $1.04(0.77-1.52)$ & $1.37(0.88-1.97)$ & $<0.01$ \\
\hline Smoking (\%) & $38(4.2)$ & $47(5.2)$ & $80(8.9)$ & $123(13.7)$ & $<0.01$ \\
\hline Diabetes (\%) & $1(0.1)$ & $3(0.3)$ & $20(2.2)$ & $72(8.0)$ & $<0.01$ \\
\hline
\end{tabular}

Data are the mean $\pm S D$, number (percentage), or median (interquartile range)

$S B P$ systolic blood pressure, DBP diastolic blood pressure, $B M I$ body mass index, $F P G$ fasting plasma glucose, $T C$ total cholesterol, $T G$ triglyceride, $L D L-C$ low-density lipoprotein cholesterol, $H D L-C$ high-density lipoprotein cholesterol, TyG triglyceride glucose, HOMA-IR homeostasis model assessment of insulin resistance 
Table 2 Correlation between the baPWV and clinical variables

\begin{tabular}{lrc}
\hline & $r$ & $P$ value \\
\hline Age & 0.507 & $<0.01$ \\
SBP & 0.355 & $<0.01$ \\
DBP & 0.329 & $<0.01$ \\
BMI & 0.062 & $<0.01$ \\
TC & 0.039 & $<0.01$ \\
LDL-C & 0.047 & $<0.01$ \\
HDL-C & -0.085 & $<0.01$ \\
TG/HDL-C & 0.106 & $<0.01$ \\
LDL-C/HDL-C & 0.079 & $<0.01$ \\
Non HDL-C & 0.071 & $<0.01$ \\
HOMA-IR & 0.188 & $<0.01$ \\
TyG index & 0.189 & $<0.01$ \\
\hline
\end{tabular}

HOMA-IR data were log-transformed because of their non-normal distribution PWV pulse wave velocity, SBP systolic blood pressure, DBP diastolic blood pressure, $B M I$ body mass index, TC total cholesterol, $L D L-C$ low-density lipoprotein cholesterol, HDL-C high-density lipoprotein cholesterol, TG triglyceride, HOMA-IR homeostasis model assessment of insulin resistance, TyG triglyceride glucose

into quartiles and using the first quartile as a reference. Based on age-adjusted multivariable logistic regression analysis, with Q1 set as the reference, the TyG index levels for Q2, Q3, and Q4 were associated with increased ORs for high baPWV in all subjects. Both in men and in women, this relationship remained statistically significant after adjusting for confounding variables. In addition, higher HOMA-IR quartiles were significantly associated with increasing baPWV, and this relationship remained significant even after adjusting for metabolic variables.
After adjusting for multiple risk factors, the OR (95\% CI) for increased baPWV in the highest HOMA-IR quartile was $1.80(1.17-2.78)$ in men and $1.46(1.06-2.47)$ in women, whereas the OR of the TyG index in the highest quartile was $2.92(1.92-4.44)$ in men and 1.84 (1.15-2.96) in women, respectively (Table 3 ).

\section{Discussion}

In this study, we identified a significant association between the TyG index and baPWV as a marker of arterial stiffness. To the best of our knowledge, this is the first study to demonstrate such a relationship between the TyG index and arterial stiffness in the general population. Furthermore, our results showed that the TyG index is independently and more strongly associated with increased arterial stiffness compared to HOMA-IR after adjusting for metabolic risk factors.

The TyG index which is a surrogate marker of IR, is known to be associated with metabolic parameters and CVD [12, 13]. Furthermore, in recent studies, it has been proven that the TyG index had some prognostic value to predict type 2 diabetes mellitus in normoglycemic patients [18] and normal-weight patients [19]. Our study showed that most of the metabolic parameters were increased or decreased according to TyG index quartiles, as expected. These results were consistent with those of previous studies [14, 20,21]. Moreover, we observed a significant correlation between the TyG index and baPWV.

A few reports have investigated whether the TyG index can serve as an independent predictor of subclinical atherosclerosis in both the general population as well as in diabetic subjects $[11,14,15]$. However, to date, only one

Table 3 Odds ratios and 95\% confidence intervals for high baPWV according to TyG index and HOMA-IR

\begin{tabular}{|c|c|c|c|c|c|}
\hline & \multicolumn{4}{|l|}{ OR $(95 \% \mathrm{Cl})$} & \multirow[t]{2}{*}{$P$ for trend } \\
\hline & Q1 (lowest) & Q2 & Q3 & Q4 (highest) & \\
\hline \multicolumn{6}{|c|}{ TyG index in men } \\
\hline Model 1 & 1.00 & $1.47(0.99-2.18)$ & $1.55(1.07-2.26)$ & $2.31(1.60-3.33)$ & $<0.01$ \\
\hline Model 2 & 1.00 & $1.55(1.02-2.36)$ & $1.81(1.20-2.71)$ & $2.92(1.92-4.44)$ & $<0.01$ \\
\hline \multicolumn{6}{|c|}{ TyG index in women } \\
\hline Model 1 & 1.00 & $1.30(0.85-1.99)$ & $2.00(1.33-3.01)$ & $2.35(1.34-4.64)$ & $<0.01$ \\
\hline Model 2 & 1.00 & $1.28(0.80-2.03)$ & $1.56(0.97-2.52)$ & $1.84(1.15-2.96)$ & $<0.01$ \\
\hline \multicolumn{6}{|c|}{ HOMA-IR in men } \\
\hline Model 1 & 1.00 & $1.24(0.85-1.83)$ & $1.39(0.95-2.03)$ & $2.15(1.48-3.11)$ & $<0.01$ \\
\hline Model 2 & 1.00 & $1.19(0.79-1.78)$ & $1.32(0.90-1.99)$ & $1.80(1.17-2.78)$ & $<0.05$ \\
\hline \multicolumn{6}{|c|}{ HOMA-IR in women } \\
\hline Model 1 & 1.00 & $1.12(0.87-1.65)$ & $1.38(0.97-1.85)$ & $2.13(1.19-3.79)$ & $<0.01$ \\
\hline Model 2 & 1.00 & $1.09(0.85-1.38)$ & $1.31(0.88-2.01)$ & $1.46(1.06-2.47)$ & $<0.05$ \\
\hline
\end{tabular}

Model 1: adjusted for age

Model 2: adjusted for age, SBP, BMI, LDL-C, HDL-C, diabetes mellitus, and menopause (women) 
study has examined the relationship between the TyG index and arterial stiffness. Lambrinoudaki et al. showed an association between the TyG index and arterial stiffness as measured using the PWV between the common carotid artery and common femoral artery [16], but this study was small in size and conducted with only postmenopausal women. However, in the present study, which was conducted in a relatively large number of healthy adults, we demonstrated that the TyG index was independently associated with increased PWV. Compared with HOMA-IR, we found that the TyG index was better at predicting increased arterial stiffness, which was consistent with previous findings showing that the TyG index is more strongly associated with carotid atherosclerosis and the prevalence of CAC than HOMA-IR $[14,15]$.

Although the mechanism underlying the relationship between TyG index and arterial stiffness has not been fully elucidated, it may be linked to IR. Indeed, previous studies have demonstrated that HOMA-IR, which reflects insulin resistance, is related to PWV [22-24]. Both the TyG index and HOMA-IR are well known representative markers of IR, and they are closely related to each other. However, our findings showed that the TyG index was better associated with arterial stiffness than HOMA-IR, which may be explained by the fact that the two indices reflect different aspects of IR. Specifically, the TyG index reflects IR in the muscle [25, 26], while HOMA-IR reflects IR in the liver [6, 27]. Thus, the TyG index may better reflect peripheral insulin resistance and may be a useful marker of arterial stiffness, including carotid and coronary atherosclerosis $[14,15]$.

Our study had several limitations. First, this was a cross-sectional observational study. Thus, a causal relationship cannot be established based on the results of this study. Second, the study population consisted of Korean men and women enrolled at a single institution, and the proportions of young adults and pre-menopausal women were relatively small. Thus, the generalizability of the results may be limited. Lastly, we were unable to obtain histories of alcohol consumption and smoking in a quantitative manner, and we could not adjust for nutritional and exercise habits, which can affect blood triglyceride levels.

\section{Conclusions}

We demonstrated a significant association between the TyG index and baPWV as a marker of arterial stiffness in both sexes. Moreover, the TyG index was found to be independently associated with increased arterial stiffness to a greater degree than was HOMA-IR. As a marker of insulin resistance, the TyG index is a simple to calculate, and it appears to be a useful marker of arterial stiffness and thus reflective of cardiovascular risk. Further prospective large-scale studies will be needed to elucidate the exact mechanism of the relationship between the TyG index and arterial stiffness.

\begin{abstract}
Abbreviations
TyG: triglyceride glucose; HOMA-IR: homeostasis model assessment of insulin resistance; PWV: pulse wave velocity; CVD: cardiovascular disease; IR: insulin resistance; ABI: ankle-brachial index; BMI: body mass index; SBP: systolic blood pressure; DBP: diastolic blood pressure; BP: blood pressure; FPG: fasting plasma glucose; TC: total cholesterol; HDL-C: high-density lipoprotein cholesterol; LDLC: low-density lipoprotein cholesterol; TG: triglycerides; SD: standard deviation; OR: odds ratio.
\end{abstract}

\section{Authors' contributions \\ JSP and SBL designed the study. CWA, BKL, SAK, JSN, JHY, MJK and MKK assisted with data acquisition and interpretation. SBL and JSP performed the statistical analysis. CWA, BKL, and JSN contributed to the discussion. SBL and JSP drafted the manuscript, and JSP revised the manuscript. All authors read and approved the final manuscript.}

\section{Author details}

${ }^{1}$ Division of Endocrinology, Department of Internal Medicine, Yonsei University College of Medicine, Gangnam Severance Hospital, 211, Eonju-ro, Gangnam-gu, Seoul, South Korea. ${ }^{2}$ Severance Institute for Vascular and Metabolic Research, Yonsei University College of Medicine, Seoul, South Korea.

${ }^{3}$ Division of Cardiology, Department of Internal Medicine, Yonsei University College of Medicine, Seoul, South Korea. ${ }^{4}$ Division of Endocrinology, Department of Internal Medicine, Hallym University Kangdong Sacred Heart Hospital, 150, Seongan-ro, Gangdong-gu, Seoul, South Korea.

\section{Acknowledgements}

We would like to thank the Gangnam Severance Health Promotion Research team for supporting the construction of the registry of data from the Health promotion center of Gangnam Severance hospital.

\section{Competing interests}

The authors declare that they have no competing interests.

\section{Availability of data and materials}

The datasets used and/or analyzed in the current study are available from the corresponding author upon reasonable request.

\section{Consent for publication}

Not applicable.

Ethics approval and consent to participate

Not applicable.

Funding

Not applicable.

\section{Publisher's Note}

Springer Nature remains neutral with regard to jurisdictional claims in published maps and institutional affiliations.

Received: 16 January 2018 Accepted: 19 March 2018

Published online: 21 March 2018

\section{References}

1. World Health Organization. Fact sheets-cardiovascular diseases. 2017. http://www.who.int/mediacentre/factsheets/fs317/en. Accessed 15 Jan 2018.

2. Sheng CS, Li Y, Li LH, Huang QF, Zeng WF, Kang YY, Zhang L, Liu M, Wei FF, Li GL, et al. Brachial-ankle pulse wave velocity as a predictor of mortality in elderly Chinese. Hypertension (Dallas, Tex: 1979). 2014;64(5):1124-30. 
3. Lillioja S, Mott DM, Spraul M, Ferraro R, Foley JE, Ravussin E, Knowler WC, Bennett PH, Bogardus C. Insulin resistance and insulin secretory dysfunction as precursors of non-insulin-dependent diabetes mellitus. Prospective studies of Pima Indians. N Eng J Med. 1993;329(27):1988-92.

4. Salonen JT, Lakka TA, Lakka HM, Valkonen VP, Everson SA, Kaplan GA. Hyperinsulinemia is associated with the incidence of hypertension and dyslipidemia in middle-aged men. Diabetes. 1998:47(2):270-5.

5. Wang F, Han L, Hu D. Fasting insulin, insulin resistance and risk of hypertension in the general population: a meta-analysis. Clin Chim Acta. 2017:464:57-63

6. Bonora E, Targher G, Alberiche M, Bonadonna RC, Saggiani F, Zenere MB, Monauni T, Muggeo M. Homeostasis model assessment closely mirrors the glucose clamp technique in the assessment of insulin sensitivity: studies in subjects with various degrees of glucose tolerance and insulin sensitivity. Diabetes Care. 2000;23(1):57-63.

7. Guerrero-Romero F, Simental-Mendia LE, Gonzalez-Ortiz M, MartinezAbundis E, Ramos-Zavala MG, Hernandez-Gonzalez SO, Jacques-Camarena O, Rodriguez-Moran $\mathrm{M}$. The product of triglycerides and glucose, a simple measure of insulin sensitivity. Comparison with the euglycemichyperinsulinemic clamp. J Clin Endocrinol Metabol. 2010;95(7):3347-51.

8. Vasques AC, Novaes FS, de Oliveira Mda S, Souza JR, Yamanaka A, Pareja JC, Tambascia MA, Saad MJ, Geloneze B. TyG index performs better than HOMA in a Brazilian population: a hyperglycemic clamp validated study. Diabetes Res Clin Pract. 2011;93(3):e98-100.

9. Mohd Nor NS, Lee S, Bacha F, Tfayli H, Arslanian S. Triglyceride glucose index as a surrogate measure of insulin sensitivity in obese adolescents with normoglycemia, prediabetes, and type 2 diabetes mellitus: comparison with the hyperinsulinemic-euglycemic clamp. Pediatric diabetes. 2016:17(6):458-65.

10. Song do K, Lee H, Sung YA, Oh JY. Triglycerides to high-density lipoprotein cholesterol ratio can predict impaired glucose tolerance in young women with polycystic ovary syndrome. Yonsei Med J. 2016:57(6):1404-11.

11. Lee EY, Yang HK, Lee J, Kang B, Yang Y, Lee SH, Ko SH, Ahn YB, Cha BY, Yoon $\mathrm{KH}$, et al. Triglyceride glucose index, a marker of insulin resistance, is associated with coronary artery stenosis in asymptomatic subjects with type 2 diabetes. Lipids Health Dis. 2016;15(1):155.

12. Sanchez-Inigo L, Navarro-Gonzalez D, Fernandez-Montero A, PastranaDelgado J, Martinez JA. The TyG index may predict the development of cardiovascular events. Eur J Clin Invest. 2016:46(2):189-97.

13. Vega GL, Barlow CE, Grundy SM, Leonard D, DeFina LF. Triglyceride-tohigh-density-lipoprotein-cholesterol ratio is an index of heart disease mortality and of incidence of type 2 diabetes mellitus in men. $J$ Investig Med. 2014:62(2):345-9.

14. Kim MK, Ahn CW, Kang S, Nam JS, Kim KR, Park JS. Relationship between the triglyceride glucose index and coronary artery calcification in Korean adults. Cardiovasc Diabetol. 2017;16(1):108.

15. Irace C, Carallo C, Scavelli FB, De Franceschi MS, Esposito T, Tripolino C, Gnasso A. Markers of insulin resistance and carotid atherosclerosis.
A comparison of the homeostasis model assessment and triglyceride glucose index. Int J Clin Pract. 2013;67(7):665-72.

16. Lambrinoudaki I, Kazani MV, Armeni E, Georgiopoulos G, Tampakis K, Rizos D, Augoulea A, Kaparos G, Alexandrou A, Stamatelopoulos K. The Ty $G$ index as a marker of subclinical atherosclerosis and arterial stiffness in lean and overweight postmenopausal women. Heart Lung Circ. 2017. https://doi.org/10.1016/j.hlc.2017.05.142.

17. Yamashina A, Tomiyama H, Takeda K, Tsuda H, Arai T, Hirose K, Koji Y, Hori S, Yamamoto Y. Validity, reproducibility, and clinical significance of noninvasive brachial-ankle pulse wave velocity measurement. Hypertens Res. 2002:25(3):359-64.

18. Navarro-Gonzalez D, Sanchez-Inigo L, Pastrana-Delgado J, FernandezMontero A, Martinez JA. Triglyceride-glucose index (TyG index) in comparison with fasting plasma glucose improved diabetes prediction in patients with normal fasting glucose: the vascular-metabolic CUN cohort. Prev Med. 2016;86:99-105.

19. Zhang M, Wang B, Liu Y, Sun X, Luo X, Wang C, Li L, Zhang L, Ren Y, Zhao Y, et al. Cumulative increased risk of incident type 2 diabetes mellitus with increasing triglyceride glucose index in normal-weight people: the rural Chinese cohort study. Cardiovasc Diabetol. 2017;16(1):30

20. Di Angelantonio E, Sarwar N, Perry P, Kaptoge S, Ray KK, Thompson A, Wood AM, Lewington S, Sattar N, Packard CJ, et al. Major lipids, apolipoproteins, and risk of vascular disease. JAMA. 2009;302(18):1993-2000.

21. Dawber TR, Kannel WB, Lyell LP. An approach to longitudinal studies in a community: the Framingham study. Ann NY Acad Sci. 1963;107:539-56.

22. Nakanishi N, Shiraishi T, Wada M. Brachial-ankle pulse wave velocity and metabolic syndrome in a Japanese population: the Minoh study. Hypertens Res. 2005;28(2):125-31.

23. Webb DR, Khunti K, Silverman R, Gray LJ, Srinivasan B, Lacy PS, Williams B, Davies MJ. Impact of metabolic indices on central artery stiffness: independent association of insulin resistance and glucose with aortic pulse wave velocity. Diabetologia. 2010;53(6):1190-8.

24. Park JS, Nam JS, Cho MH, Yoo JS, Ahn CW, Jee SH, Lee HS, Cha BS, Kim $\mathrm{KR}$, Lee HC. Insulin resistance independently influences arterial stiffness in normoglycemic normotensive postmenopausal women. Menopause. 2010;17(4):779-84.

25. Han T, Cheng Y, Tian S, Wang L, Liang X, Duan W, Na L, Sun C. Changes in triglycerides and high-density lipoprotein cholesterol may precede peripheral insulin resistance, with 2 -h insulin partially mediating this unidirectional relationship: a prospective cohort study. Cardiovasc Diabetol. 2016;15(1):154

26. Riboldi BP, Luft VC, de Castilhos CD, de Cardoso LO, Schmidt MI, Barreto SM, de Sander MF, Alvim SM, Duncan BB. Glucose and triglyceride excursions following a standardized meal in individuals with diabetes: ELSABrasil study. Cardiovasc Diabetol. 2015;14:21.

27. Tripathy D, Almgren P, Tuomi T, Groop L. Contribution of insulin-stimulated glucose uptake and basal hepatic insulin sensitivity to surrogate measures of insulin sensitivity. Diabetes Care. 2004;27(9):2204-10.

\section{Submit your next manuscript to BioMed Central and we will help you at every step:}

- We accept pre-submission inquiries

- Our selector tool helps you to find the most relevant journal

- We provide round the clock customer support

- Convenient online submission

- Thorough peer review

- Inclusion in PubMed and all major indexing services

- Maximum visibility for your research

Submit your manuscript at www.biomedcentral com/submit
BioMed Central 